\title{
O gênero Paradiacheopsis (Stemonitales, Myxomycetes) no Brasil
}

\author{
Andrea Carla Caldas Bezerra ${ }^{1,3}$ e Laise de Holanda Cavalcanti ${ }^{2}$
}

Recebido em 5/11/2007. Aceito em 4/06/2008

\begin{abstract}
RESUMO - (O gênero Paradiacheopsis (Stemonitales, Myxomycetes) no Brasil). Levantamento da diversidade de mixomicetos no Parque Estadual das Dunas do Natal, Unidade de Conservação de Mata de Duna Litorânea no Rio Grande do Norte e parte da Reserva da Biosfera da Mata Atlântica Brasileira, resultou no registro de Paradiacheopsis longipes Hoof \& Nann.-Bremek., nova ocorrência para o Brasil e segunda para a Região Neotropical. Além de $P$. curitibana Hertel, conhecida apenas na localidade tipo, em Curitiba, PR, não se dispunha de registro de ocorrência de representantes do gênero para o Brasil. P. longipes é ilustrada e comentada quanto aos caracteres taxonômicos mais relevantes.
\end{abstract}

Palavras-chave: mixomicetos, Neotrópicos, Stemonitaceae, taxonomia

ABSTRACT - (The genus Paradiacheopsis (Stemonitales, Myxomycetes) in Brazil). Slime molds diversity survey in the Parque Estadual das Dunas do Natal, a conservation area of coastal dune forest in Rio Grande do Norte and part of Biosphere reserve of Brazilian Atlantic Forest, resulted on the recording of Paradiacheopsis longipes Hoof \& Nann.-Bremek., new occurrence for Brazil and second for the neotropical region. Besides P. curitibana Hertel, only known in the locality type, in Curitiba, PR, this genus was not registered before in Brazil. P. longipes is illustrated and comments about the most relevant taxonomic characters are made.

Key words: Neotropics, Slime mold, Stemonitaceae, taxonomy

\section{Introdução}

Paradiacheopsis Hertel apresenta como característica principal, que o distingue dos demais gêneros de Stemonitales, um capilício ramificado, sem formar rede ou anastomoses, associado a um fibroso, com base de coloração vermelho-amarelada ou alaranjada (Nannenga-Bremekamp 1967). No artigo onde foi proposto o gênero (Hertel 1954) encontra-se a diagnose de $P$. curitibana Hertel, conhecida apenas do material tipo, coletado em Curitiba, PR, o qual foi mantido na coleção pessoal de R.J.G. Hertel, e perdido após o seu falecimento em 1985. Esta espécie é incluída por Farr (1976) entre os taxa duvidosos e/ou excluídos, listados em sua Monografia para a região Neotropical. A referida autora a considera como uma possível Comatricha elegans (Racib.) G. Lister, por apresentar em sua descrição a estrutura do pedicelo fibroso, ausência de columela, perídio evanescente, capilício com ramificações espaçadas, partindo da base do esporângio e esporos equinulados, com 10,5 $\mu$ m diâm. Vale salientar que Farr não examinou o espécime coletado por Hertel e na descrição original da espécie não há indicação do pedicelo apresentar-se fibroso.
Na descrição de Paradiacheopsis, Hertel (1954) coloca a ausência de columela entre os caracteres típicos do gênero: "Esporângios globosos ou subglobosos; pedicelados. Perídio completamente evanescente (não visto); columela falta; filamentos capiliciais originando-se na base do esporângio por quatro ramos (dicotomia ou pseudodicotomia) do pedicelo; ramificações e anastomoses pouco numerosas; esporos violáceos (por transparência)."

Em outra publicação Hertel (1956) acrescenta novos caracteres à descrição: "Esporângios dispersos, raro com tendência gregária; globosos até curtos ovóides; pedicelados. Perídio totalmente evanescente. Pedicelo no mínimo $1 / 2$ da altura total. Columela curta, atingindo apenas $1 / 2$ ou $1 / 3$ do esporângio; mais ou menos nítida, mais ou menos cônica. Capilício originando-se quase que exclusivamente, no ápice, 'atravez' (sic) de 2-6 ou mais filamentos primários, raro também por ramificações laterais da columela (cf. Comatricha lurida). Esporos cinzentos ou pardo-violáceos; verrucosos ou espinulosos. Hipotalo mais ou menos circular e reduzido". No mesmo artigo, o autor transfere para o gênero espécies de Lamproderma $[$ L. arcyrionema Rostaf. $=$ Collaria arcyrionema (Rostaf.) Nann.-Bremek. ex Lado] e

1 Programa de Pós-graduação em Ciências Biológicas, Doutorado, Centro de Ciências Biológicas, Universidade Federal de Pernambuco

2 Universidade Federal de Pernambuco, Departamento de Botânica, Centro de Ciências Biológicas, Av. Prof. Moraes Rego s.n., Cidade Universitária, 50670-090 Recife, PE, Brasil

3 Autor para correspondência: labmix@gmail.com 
Comatricha $[$ C. cornea $\mathrm{G}$. Lister \& Cran. = Macbrideola cornea (G. Lister \& Cran.) Alexop., C. fimbriata G. Lister $\&$ Cran., C. lurida Lister $=$ Collaria lurida $($ Lister $)$ Nann.Bremek., C. elegans (Racib.) G. Lister, C. rubens Lister = Collaria rubens (Lister) Nann.-Bremek. e C. martinii Alexop. \& Beneke = Macbrideola martinii (Alexop. \& Beneke) Alexop.]. Destas, apenas P. fimbriata (G.Lister \& Cran) Hertel ex Nann.-Bremek. é válida atualmente. Dentre as espécies descritas para o gênero por E. Nannenga-Bremekamp e válidas até o momento, apenas $P$. cribrata Nann.-Bremek. e P. longipes Hoof \& Nann.-Bremek. não foram transferidas de um outro gênero.

Nannenga-Bremekamp $(1967 ; 1991)$ separa Paradiacheopsis dos demais gêneros da ordem, pela presença de um capilício ramificado, sem anastomoses e comenta que um pedicelo fibroso, de base vermelhoamarelada a alaranjada também é encontrado em Lamproderma e Comatricha. A referida autora não aceitou o gênero Comatrichoides proposto por Hertel (1956) e incluiu suas espécies em Paradiacheopsis, aceitando, entretanto, Paradiachea Hertel e as três espécies nele incluídas. Martin \& Alexopoulos (1969) não acataram inicialmente as proposições de Hertel (1956), aceitando-as após mais de uma década (Martin et al. 1983).

O gênero Paradiacheopsis compreende atualmente 10 espécies válidas e inclui oito espécies colocadas em sinonímia ou como duvidosas (Hernández-Crespo \& Lado 2005).

Recentemente, Schnittler \& Stephenson (2000) relataram a presença de $P$. longipes Hoof em florestas úmidas da Costa Rica, sem fornecer dados sobre o microhabitat nem com que frequiência e abundância a espécie foi encontrada. Provavelmente esta foi a primeira referência de $P$. longipes para a Região Neotropical, sendo conhecida anteriormente para a China (Ukkola et al. 2001), França, Holanda (van Hoof \& NannengaBremekamp 1996) e Tanzânia (Ukkola 1998).

No presente trabalho, apresenta-se o segundo registro de $P$. longipes para os Neotrópicos e o segundo de um representante de Paradiacheopsis para o Brasil, 50 anos após a proposição do gênero por Hertel (1954), quando foi descrita $P$. curitibana, restrito até o momento à Região Sul do país.

\section{Material e métodos}

O Parque Estadual das Dunas do Natal (05\% $48^{\prime}$ $05^{\circ} 53^{\prime} \mathrm{S}$ e $35^{\circ} 09^{\prime}-35^{\circ} 12^{\prime} \mathrm{W}$ ) é parte integrante da Reserva da Biosfera da Mata Atlântica Brasileira, abrangendo 1.172 ha. de mata nativa, representada pela Mata de Duna Litorânea (Freire 1990). Essa área integra os fragmentos de Floresta Atlântica analisados em outro projeto (A.C.C. Bezerra, em preparação), que visa o levantamento da diversidade de Myxomycetes em Unidades de Conservação do Rio Grande do Norte. O clima local é quente e úmido, com índices de pluviosidade elevados entre maio e julho, atingindo $1.900 \mathrm{~mm}$ anuais; as chuvas são ocasionadas, na região, pelo avanço da massa Equatorial Atlântica, começando a estação úmida entre os meses de março e abril, com pluviosidade máxima em junho, estendendo-se as chuvas até agosto/setembro (Freire 1990). Coletas de mixomicetos foram realizadas no início da estação chuvosa (maio) de 2005 e início da estiagem (setembro) de 2005 e 2006.

Amostras de substrato foram colhidas e utilizadas para cultivo em câmara-úmida, mantidas à luz e temperatura ambientes e observadas por três meses (Novozhilov et al. 2001). A análise das coleções, todas obtidas em câmara-úmida, foi realizada seguindo a metodologia descrita por Mobin \& Cavalcanti (2000). Exsicatas representativas do material estudado encontram-se depositadas no herbário UFP (Universidade Federal de Pernambuco, Departamento de Botânica). Para identificação dos espécimes foram empregados os trabalhos de Nannenga-Bremekamp (1991), Ukkola (1998) e Mitchell (2004). A descrição e as ilustrações basearam-se no material coletado, visando informar características dos espécimes que ocorrem no Brasil. A nomenclatura taxonômica e abreviatura dos nomes dos autores seguem Hernández-Crespo \& Lado (2005).

\section{Resultados e discussão}

Paradiacheopsis longipes Hoof \& Nann.-Bremek., Proc. Kon. Ned. Akad. Wetensch. 99(1-2): 51. 1996.

Fig. 1

Esporângio pedicelado, disperso, altura total $2,0 \mathrm{~mm}$; esporoteca globosa, pendente, castanhoamarelada; pedicelo $1,7 \mathrm{~mm}$ compr., fibroso, castanho brilhante por luz incidente, castanho-avermelhado escuro por luz transmitida, translúcido no ápice $(8,0 \mu \mathrm{m}$ larg.), base castanho-amarelada e alargada $(26,0 \mu \mathrm{m}$ larg.); hipotalo membranoso, pouco desenvolvido, castanhoavermelhado; perídio totalmente evanescente; columela afinando para o ápice $(4,0 \mu \mathrm{m})$, atingindo $1 / 3-1 / 2$ da esporoteca; capilício delicado (3,0 $\mu \mathrm{m}$ larg.), 2-3 ramificações primárias saindo do ápice da columela, afinando para o ápice $(1,0 \mu \mathrm{m}$ larg.), castanho claro; esporada castanha; esporo globoso, castanho claro, minutamente espinuloso, 8,0 $\mu \mathrm{m}$ diâm.

Material examinado: BRASIL. Rio Grande do Norte: Natal, Parque das Dunas do Natal. Trilha Peroba, cultivado em 25/IV/2005, esporulado em 7/VI/2005, Myrcya multiflora DC. (pau-mulato), A.C.C. Bezerra 


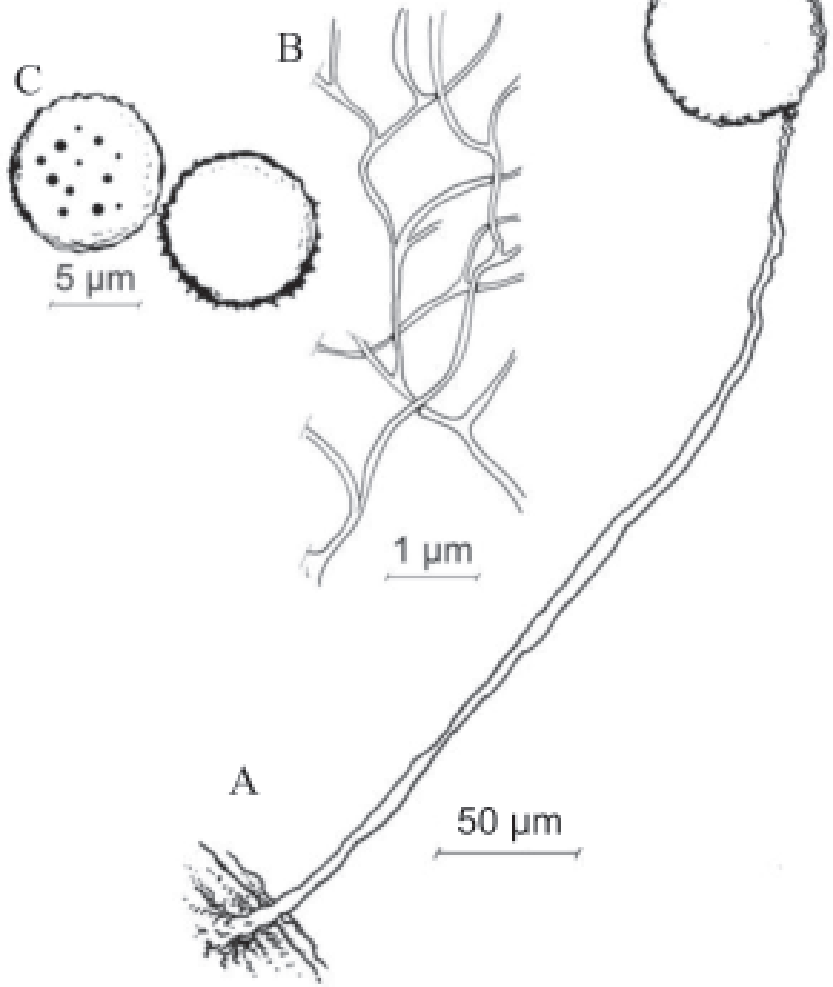

Figura 1. Paradiacheopsis longipes Hoof e Nann.-Bremek. A. Esporângio. B. Detalhe da ramificação do capilício. C. Esporos.

et al. (UFP 45132); ibid. esporulado em 12/VI/2005, Myrcya multiflora DC. (pau-mulato), A.C.C. Bezerra et al. (UFP 45133).
O material coletado no presente estudo enquadrase bem na descrição do tipo de $P$. longipes, proposta por Van Hoof \& Nannenga-Bremekamp (1996) com base em espécime desenvolvido em câmara-úmida; apresenta também semelhança com a descrição do espécime coletado na Tanzânia, a ca. de $900 \mathrm{~m}$ de altitude, descrito por Ukkola (1998).

O substrato onde o material tipo de $P$. longipes se desenvolveu em câmara-úmida pertence a um indivíduo do gênero Quercus (Fagaceae), que reúne muitas espécies arbóreas, de córtex rico em taninos, geralmente espesso nos indivíduos adultos, com fissuras profundas. Wrigley de Basanta (1998) comenta que este tipo de córtex, que se divide em placas, oferece ambiente propício ao desenvolvimento de mixomicetos e relaciona $P$. fimbriata entre as espécies reportadas na literatura para $Q$. ilex $\mathrm{L}$.

Conhecida localmente como pau-mulato, Myrcya multiflora (Myrtaceae) é uma espécie arbórea de médio porte, comum no ambiente de restinga onde $P$. longipes foi registrada, em altitude próxima ao nível do mar (4 m). Como é freqüente em espécies de Myrtaceae, o tronco do pau-mulato apresenta ritidoma que se desprende em tiras largas, delgadas, deixando exposto o tronco liso e brilhante (Fig. 2), não muito propício para o desenvolvimento de mixomicetos.

$\mathrm{O}$ registro da presença de $P$. longipes no ambiente estudado só foi possível com o auxílio da técnica de câmara-úmida, como é comum acontecer com espécies do gênero; Novozhilov et al. (2000), por exemplo, só

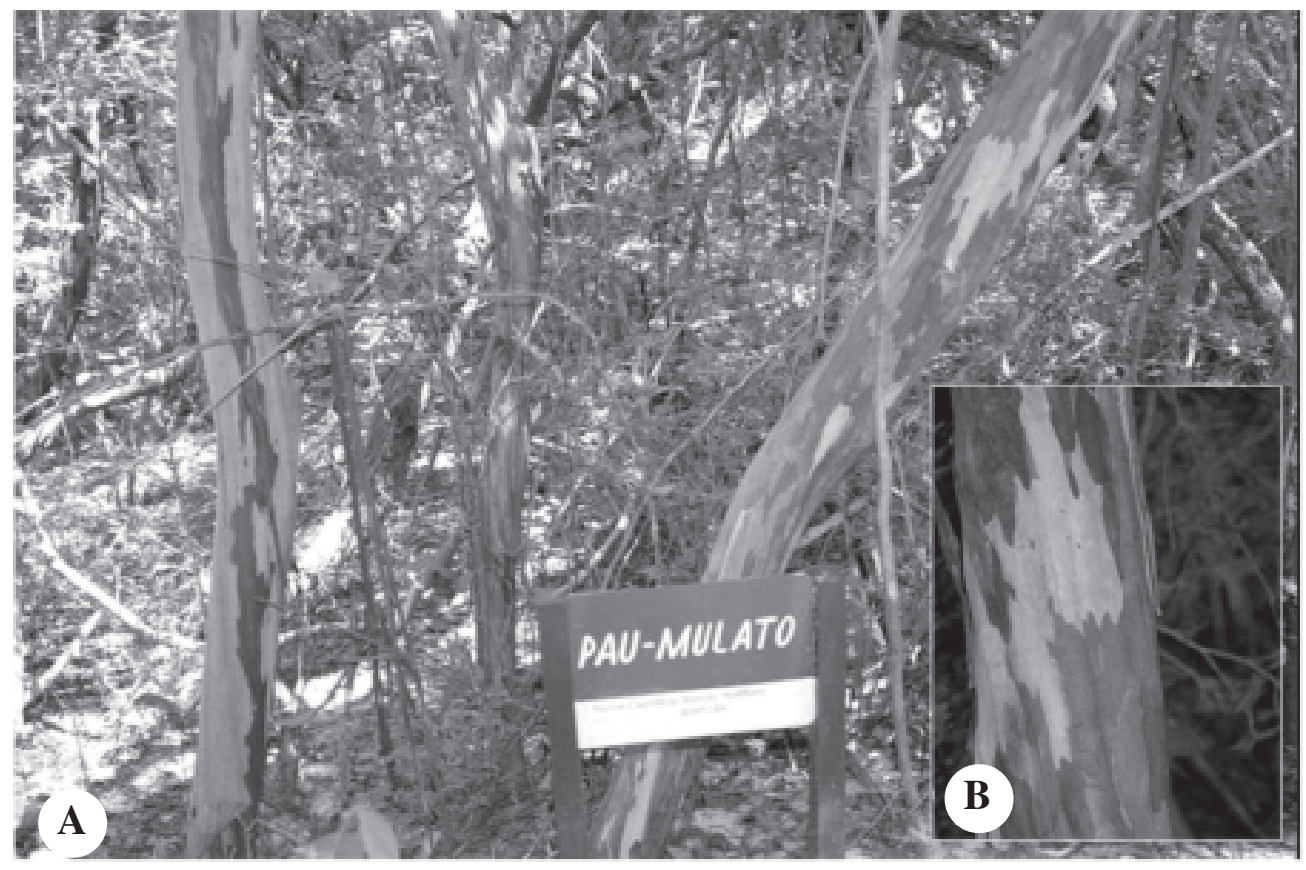

Figura 2. A. Indivíduos de Myrcya multiflora DC. (pau-mulato) presentes na Trilha Peroba do Parque das Dunas do Natal, RN, Brasil. B. Detalhe do tronco do pau-mulato, ritidoma que se desprende em tiras largas e delgadas. 
registraram a ocorrência de $P$. cribrata, $P$. solitaria e $P$. fimbriata em diferentes ambientes vegetacionais da Rússia e Casaquistão, com o recurso da câmara-úmida. Ukkola (1998) relata a ocorrência na África de P. rigida, em casca do tronco de duas espécies de Cupressaceae (Cupressus lusitanica Mill. e Juniperus virginiana L.) e $P$. longipes, em câmaras-úmidas montadas com folhas mortas não identificadas. Como novas referências para a China, Ukkola et al. (2001) registraram três espécies de Paradiacheopsis, dentre elas P. longipes, desenvolvidas em câmara-úmida de Pinus massoniana Lamb. (Pinaceae), Cryptomeria fortunei Hooibrenk ex Otto et Dietr. $=C$. japonica Thunberg ex L.f. (Cupressaceae) e Sassafras tzumu (Hemsl.) Hemsl. (Lauraceae), espécies muito importantes como fornecedoras de madeira, a primeira delas sendo também usada para obtenção de resina, tanino e para cultivar fungos no Sul da China.

Além de P. curitibana Hertel, conhecida apenas na localidade tipo, Curitiba, PR, onde foi coletada em uma propriedade particular do seu autor, não se dispunha de registro de ocorrência de representantes do gênero para o Brasil. Paradiacheopsis está, portanto, representado no país por pelo menos duas espécies, uma delas, P. curitibana, sem registros há mais de 50 anos e, que dentro dos padrões empregados pela União Internacional para a Conservação da Natureza (IUCN) para outros organismos, poderia ser considerada como exposta a um risco extremamente elevado de extinção na Natureza.

\section{Agradecimentos}

As autoras agradecem aos integrantes do Laboratório de Myxomycetes (LABMIX), pelo auxílio em campo e no laboratório, em especial a Alissandra Nunes, Aurelice Costa, David Lemos, Fátima Bezerra, Glauciane Damasceno, Inaldo Ferreira, Juciara Gouveia, Leandro Agra, Marcio Rufino e Wendell Medrado; à direção do Parque das Dunas do Natal, pelo apoio concedido durante o período de coleta; ao Conselho Nacional de Desenvolvimento Científico e Tecnológico
(CNPq), pelo suporte financeiro; ao Departamento de Botânica da Universidade Federal de Pernambuco, pelo suporte físico.

\section{Referências bibliográficas}

Farr, M.L. 1976. Flora Neotropica. Monograph 16. New York, New York Botanical Garden.

Freire, S.M.B. 1990. Levantamento florístico do Parque Estadual das Dunas do Natal. Acta Botanica Brasilica 4: 41-59.

Hernández-Crespo, J. C. \& Lado, C. 2005. An on-line nomenclatural information system of Eumycetozoa.http://www.nomen. eumycetozoa.com

Hertel, R.J.G. 1954. Myxomycetes do Brasil. II. Paradiacheopsis curitibana Hertel, n.gen. e n.sp. de Lamprodermaceae. Dusenia 5: $191-192$

Hertel, R.J.G. 1956. Taxonomia de Comatricha Preuss emend. Rost. (Myxophyta). Dusenia 7: 341-350.

Martin, G.W. \& Alexopoulos, C.J. 1969. The Myxomycetes. Iowa City, University of Iowa Press.

Martin, G.W.; Alexopoulos, C.J. \& Farr, M.L. 1983. Genera of Myxomycetes. Iowa City, University of Iowa Press.

Mitchell, D. 2004. A key to corticolous Myxomycota. Systematics and Geography of Plants 74: 261-285.

Mobin, M. \& Cavalcanti, L.H. 2000. Myxomycetes em Carnaúba (Copernicea prunifera, Arecaceae). Acta Botanica Brasilica 14: $71-75$.

Nannenga-Bremekamp, N.E. 1967. Notes on Myxomycetes. XII. A revision of the Stemonitales. Proceedings Konienklijke Nedederlandse Akademia van Weternschappen. Series C, Biological and Medical Sciences 70: 201-216.

Nannenga-Bremekamp, N.E. 1991. A guide to temperate Myxomycetes. Bristol, Biopress Limited.

Novozhilov, Y, K.; Schnittler, M.; Rollins, A.W. \& Stephenson, S.L. 2001. Myxomycetes from different forest types in Puerto Rico. Mycotaxon 77: 285-299.

Novozhilov, Y.K.; Schnittler, M.; Zemlianskaia, I.V. \& Fefelov, K.A. 2000. Biodiversity of plasmodial slime moulds (Myxogastria): measurement and interpretation. Protistology 1: $161-178$.

Schnittler, M. \& Stephenson, S.L. 2000. Myxomycete biodiversity in four different forest types in Costa Rica. Mycologia 92: 626-637.

Ukkola, T. 1998. Myxomycetes of the Usambara Mountains, northeast Tanzania. Acta Botanica Fennica 160: 1-37.

Ukkola, T.; Harkonen, M. \& Zeng, Z. 2001. Myxomycetes of Hunan Province, China. I. Annales Botanici Fenicci Helsinki 38: 305-328.

Van Hooff, J.P.M. \& Nannnega-Bremekamp, N.E. 1996. Additions to the Myxomycetes of the Netherlands. Proceedings Konienklijke Nedederlandse Akademia van Weternschappen 99: 45-53.

Wrigley de Basanta, D. 1998. Myxomycetes from the bark of the evergreen oak Quercus ilex. Anales del Jardín Botánico de Madrid 56: 3-14. 